\title{
Three-dimensional Simulations of Electron Beams Focused by Periodic Permanent Magnets
}

\author{
Carol L. Kory \\ Analex Corporation \\ NASA Lewis Research Center \\ 21000 Brookpark Road, MS 54-5 \\ Cleveland, $\mathrm{OH} 44135$ \\ (216) 433-3512 Phone \\ (216) 433-8705 Fax \\ Carol.L.Kory@lerc.nasa.gov
}

A fully three-dimensional (3D) model of an electron beam focused by a periodic permanent magnet (PPM) stack has been developed. First, the simulation code MAFIA was used to model a PPM stack using the magnetostatic solver. The exact geometry of the magnetic focusing structure was modeled; thus, no approximations were made regarding the off-axis fields. The fields from the static solver were loaded into the $3 \mathrm{D}$ particle-in-cell (PIC) solver of MAFIA where fully 3D behavior of the beam was simulated in the magnetic focusing field. The PIC solver computes the time-integration of electromagnetic fields simultaneously with the time integration of the equations of motion of charged particles that move under the influence of those fields. Fields caused by those moving charges are also taken into account; thus, effects like space charge and magnetic forces between particles are fully simulated [']. The electron beam is simulated by a number of macro-particles. These macro-particles represent a given charge $\mathrm{Q}$ amounting to that of several million electrons in order to conserve computational time and memory. Particle motion is unrestricted, so particle trajectories can cross paths and move in three dimensions under the influence of $3 \mathrm{D}$ electric and magnetic fields. Correspondingly, there is no limit on the initial current density distribution of the electron beam, nor its density distribution at any time during the simulation.

Simulation results including beam current density, percent ripple and percent transmission will be presented, and the effects current, magnetic focusing strength and thermal velocities have on beam behavior will be demonstrated using $3 \mathrm{D}$ movies showing the evolution of beam characteristics in time and space. Unlike typical beam optics models, this 3D model allows simulation of asymmetric designs such as noncircularly symmetric electrostatic or magnetic focusing as well as the inclusion of input/output couplers.

' The MAFIA Collaboration, MAFIA TS3 the 3D-PIC Solver, December, 1996. 

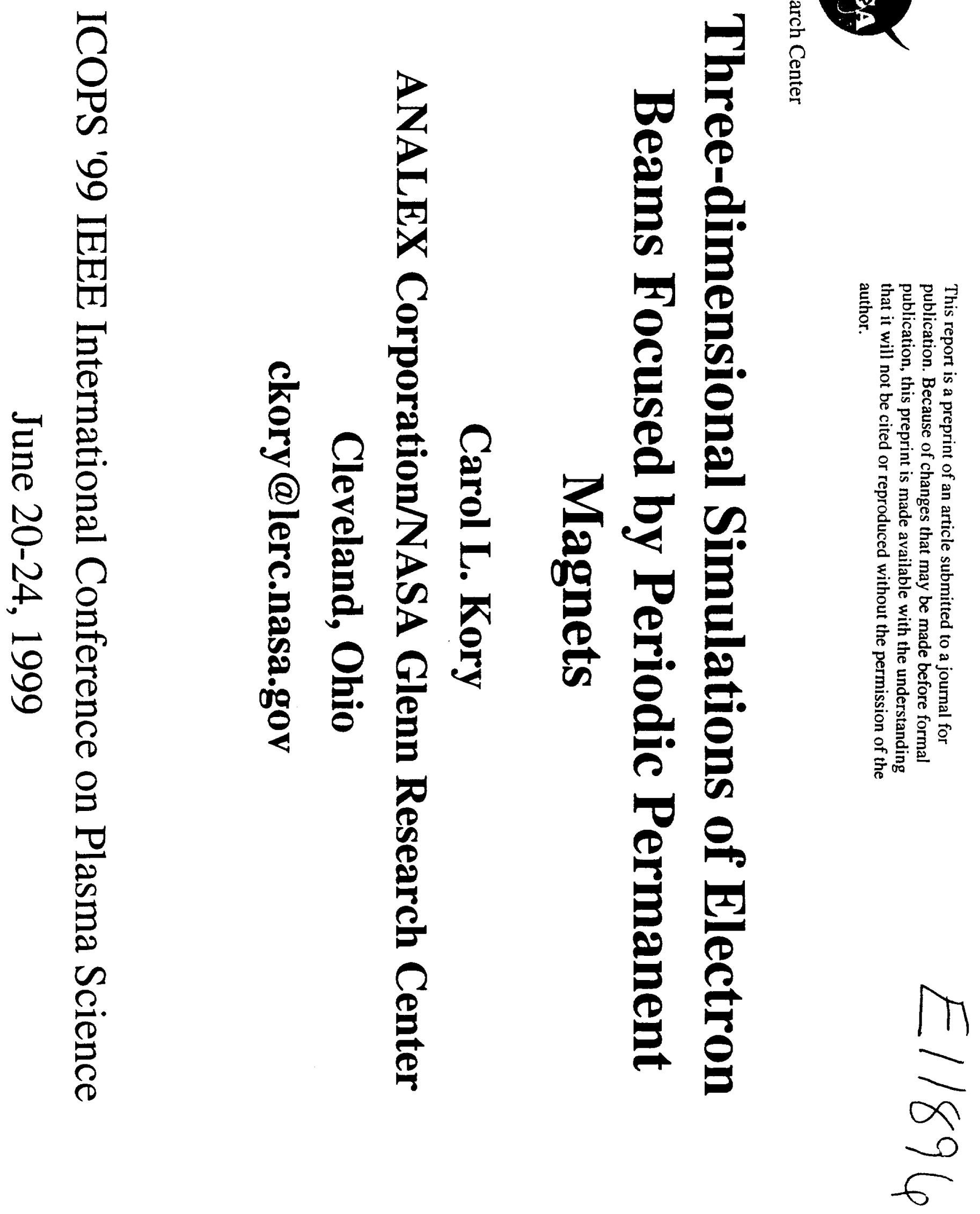


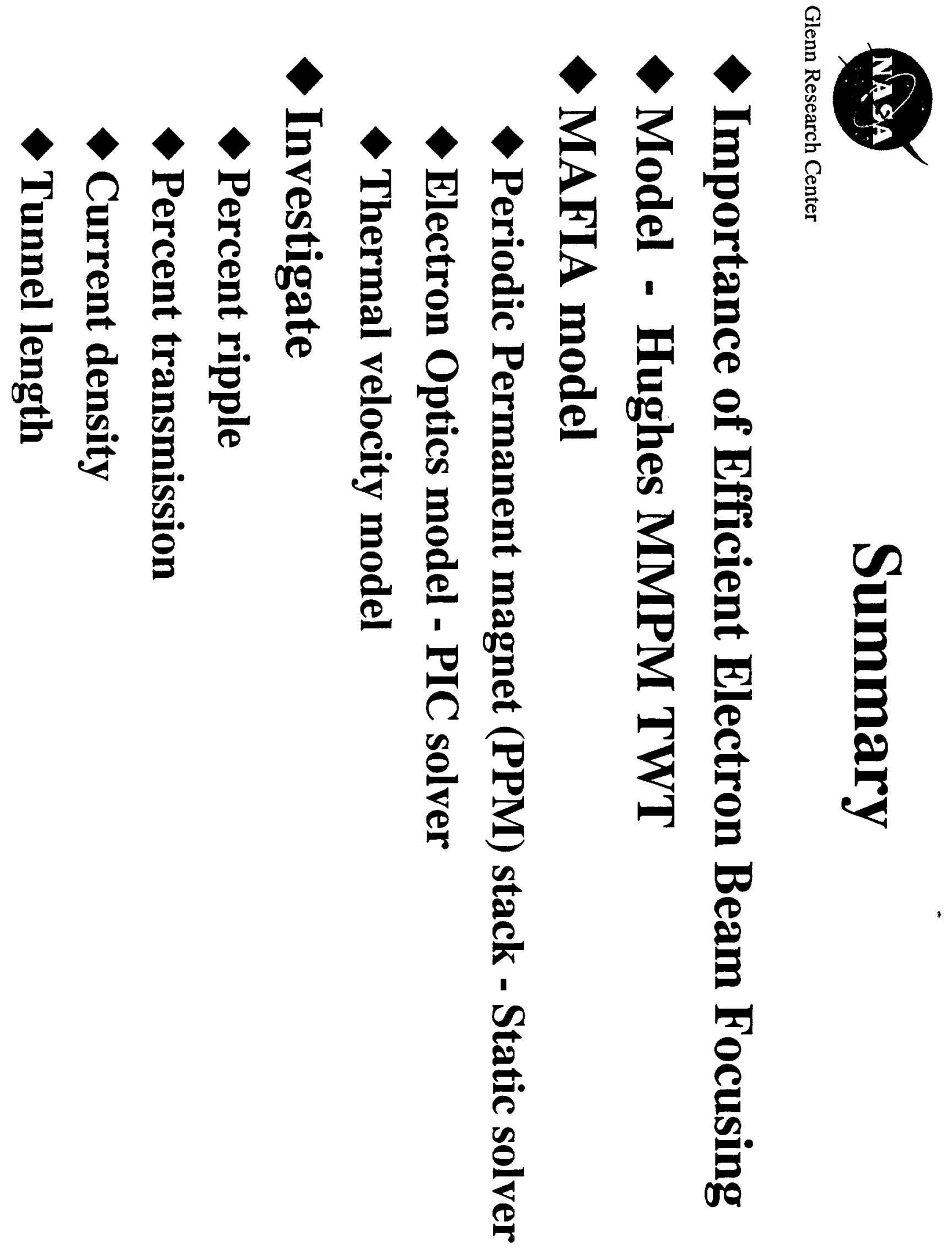




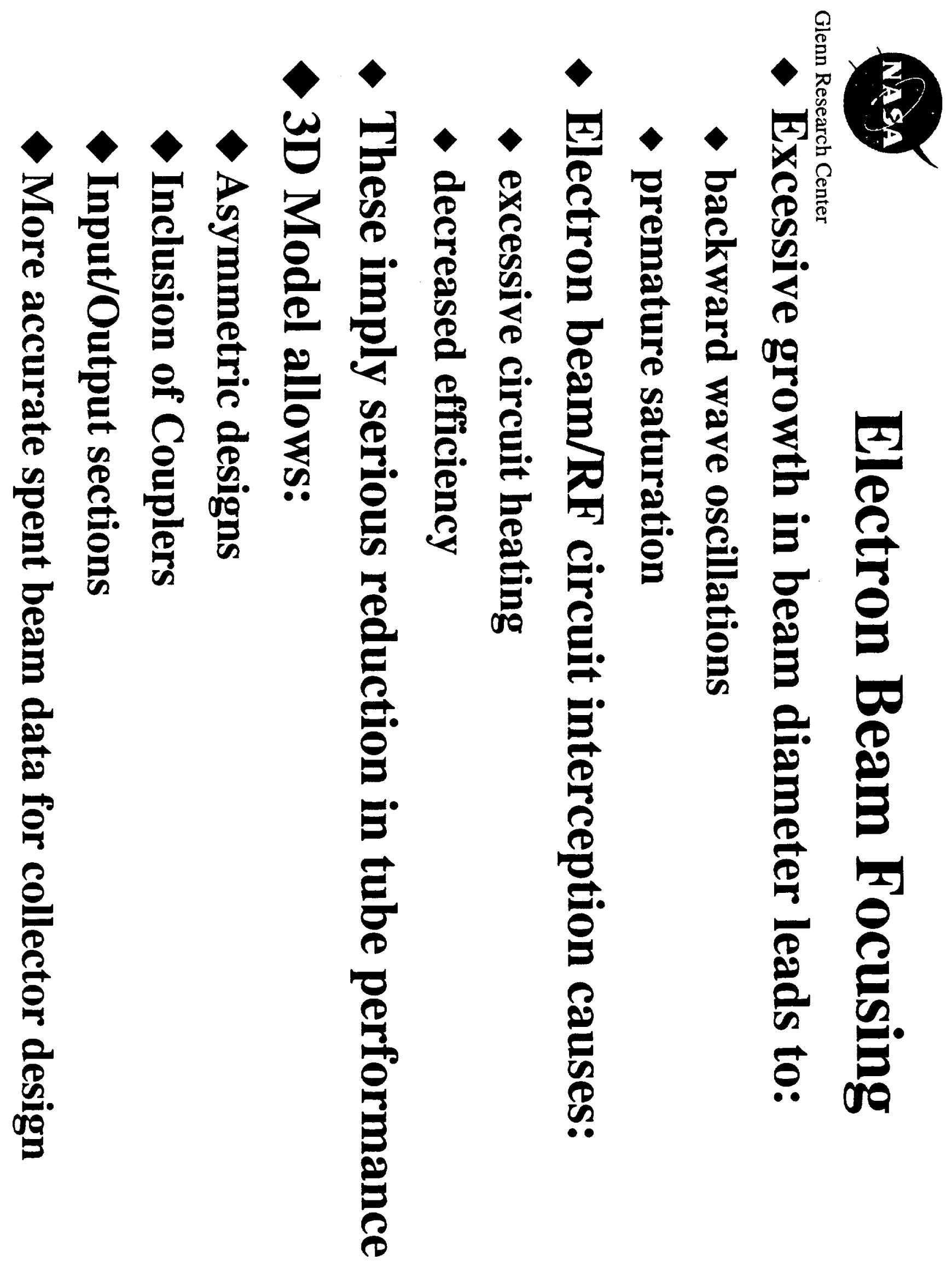



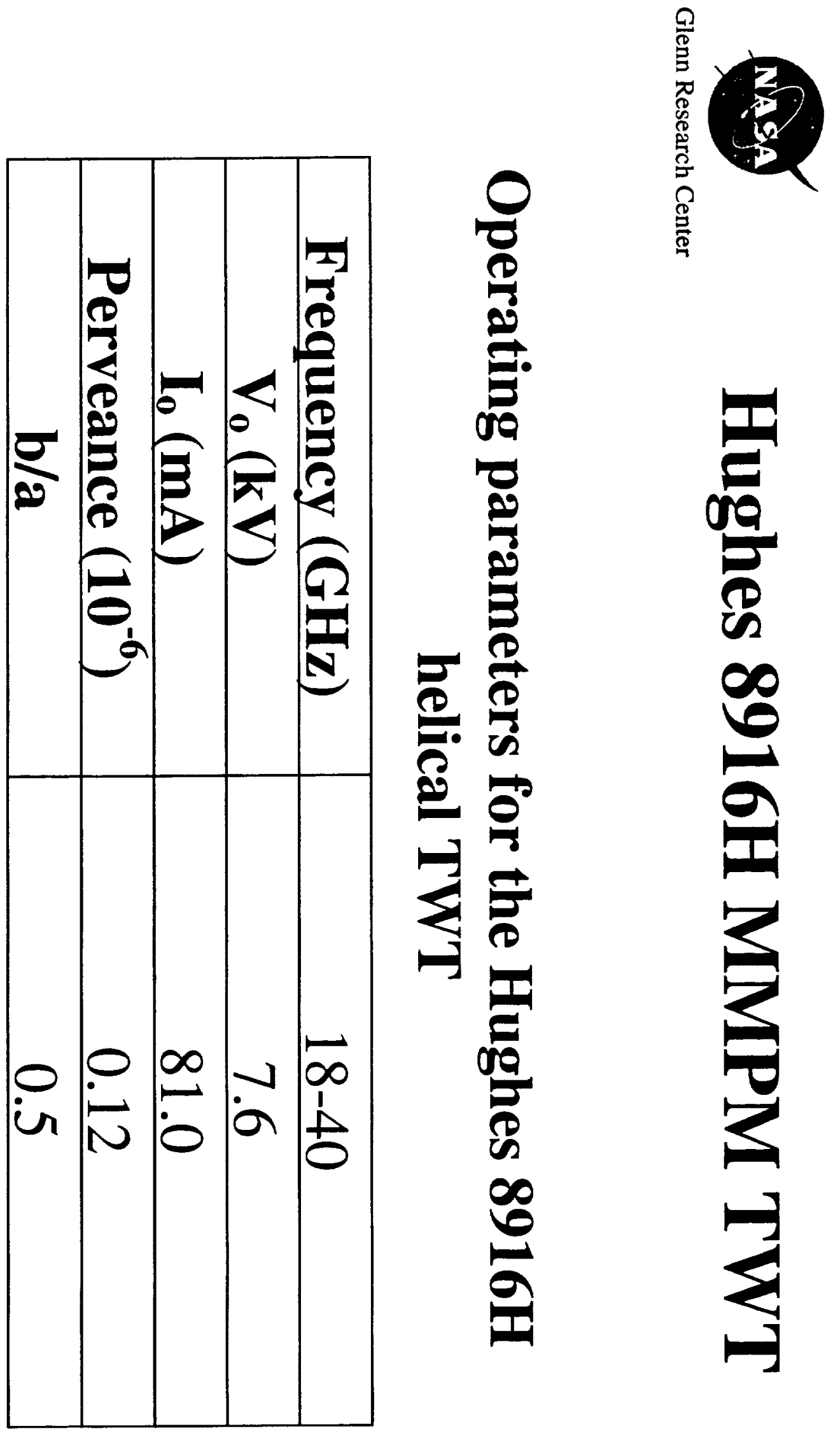


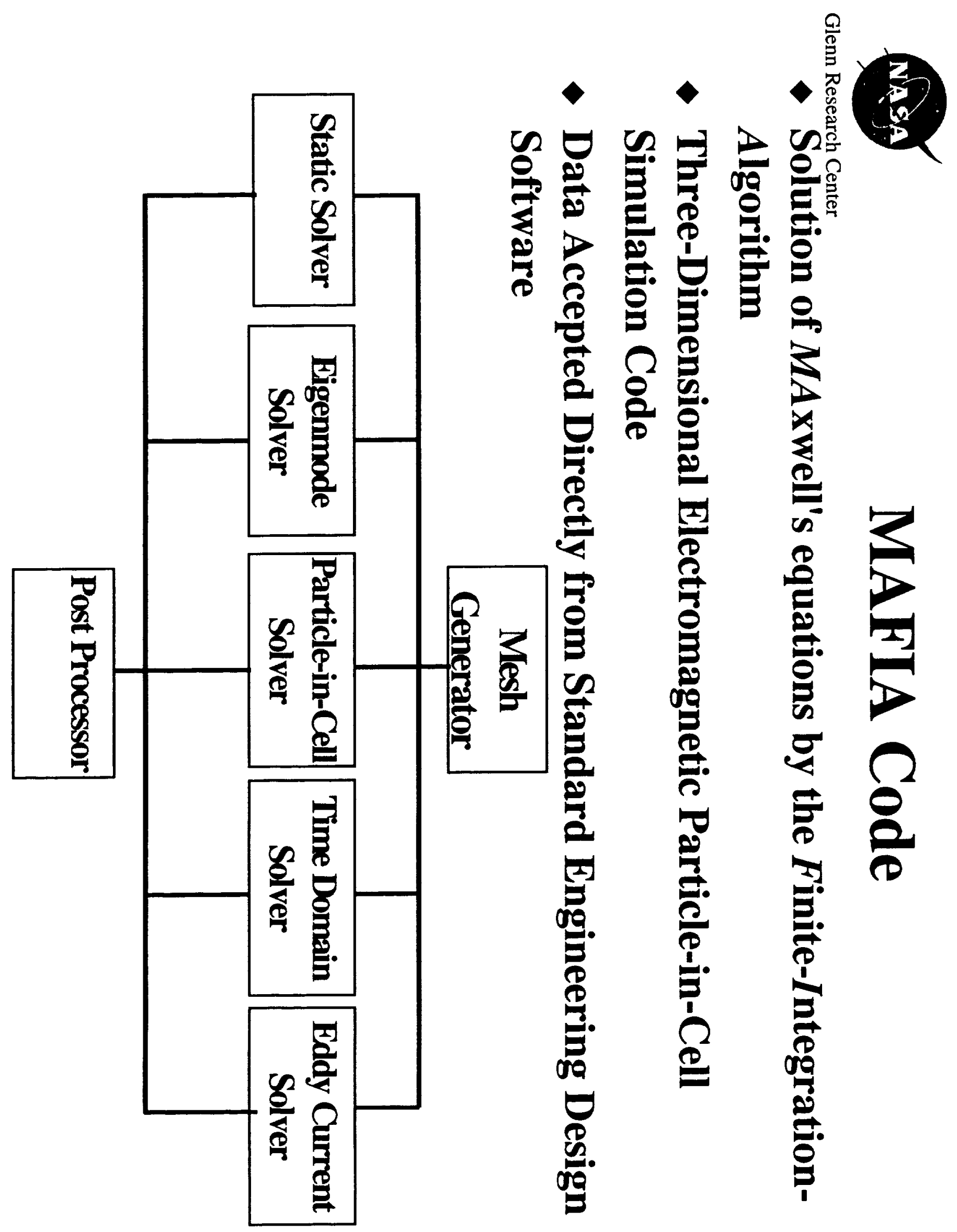




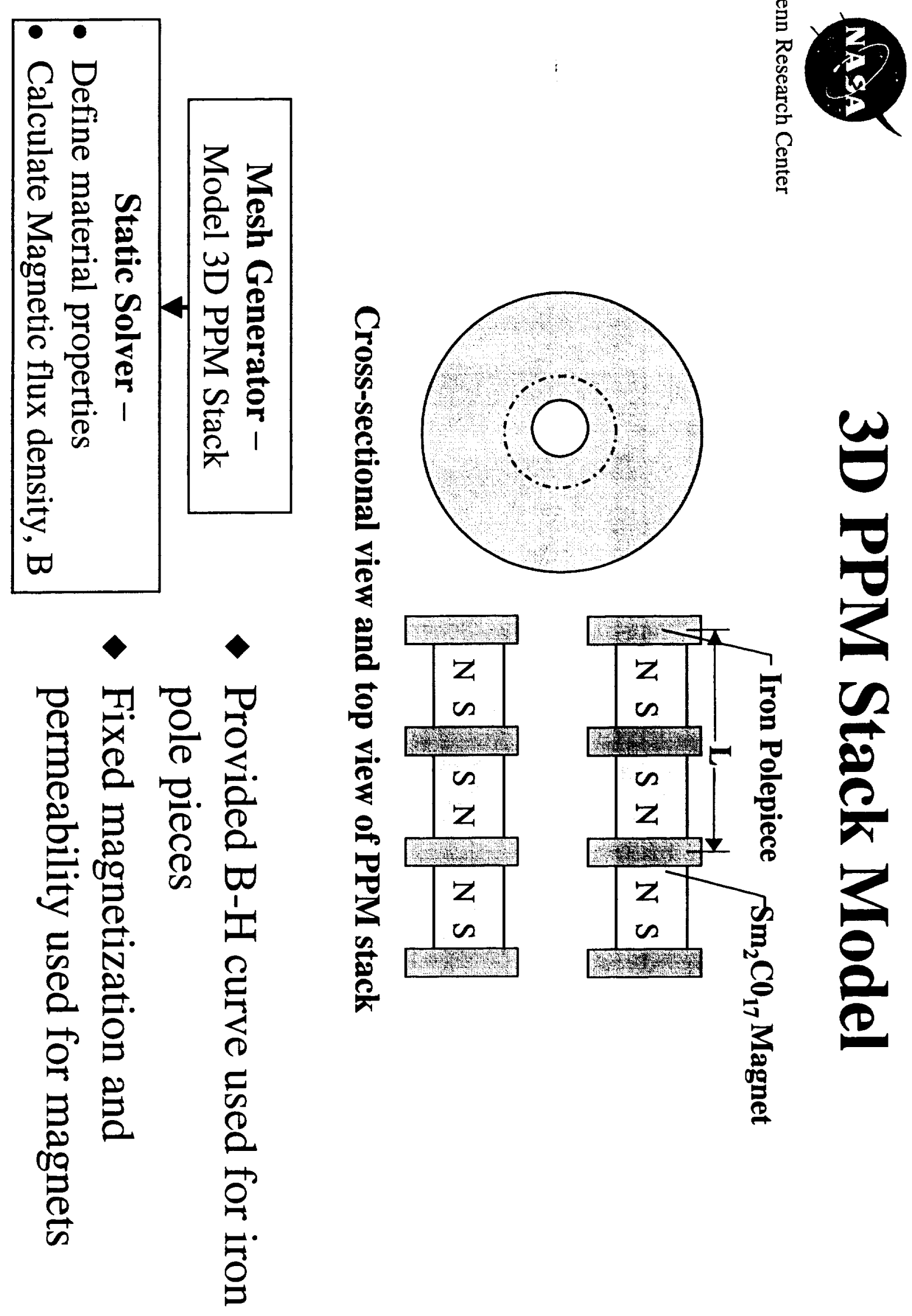



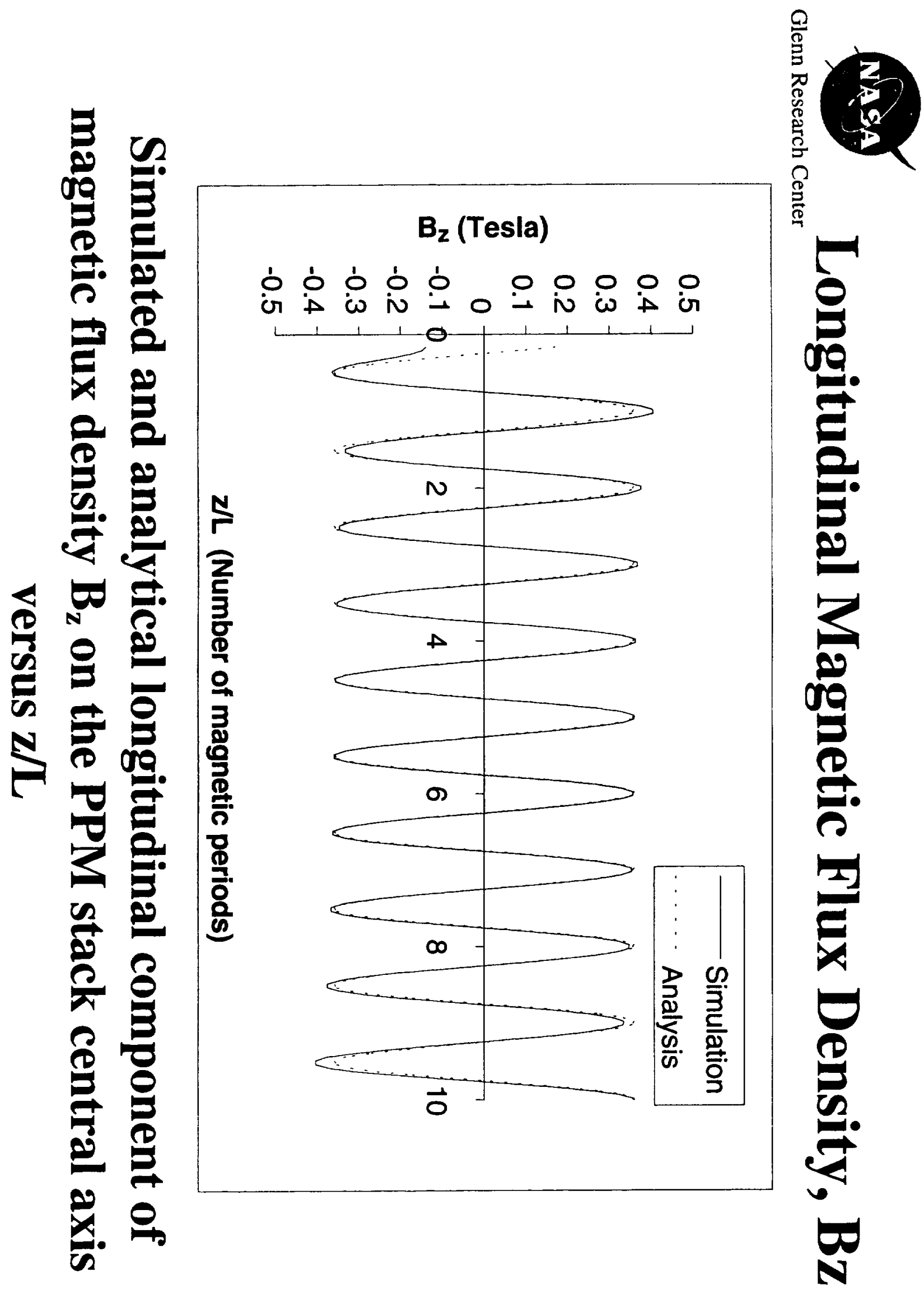


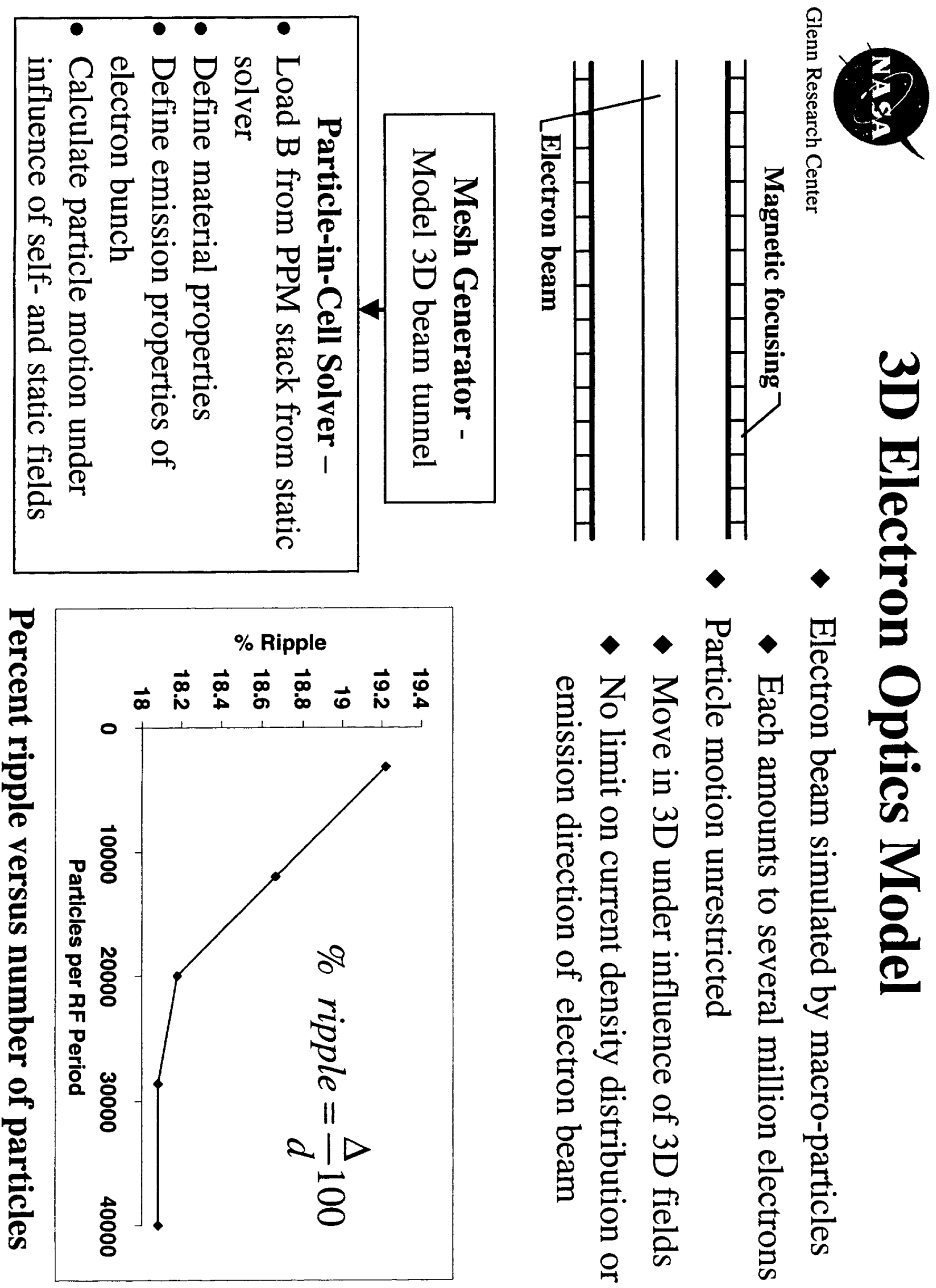




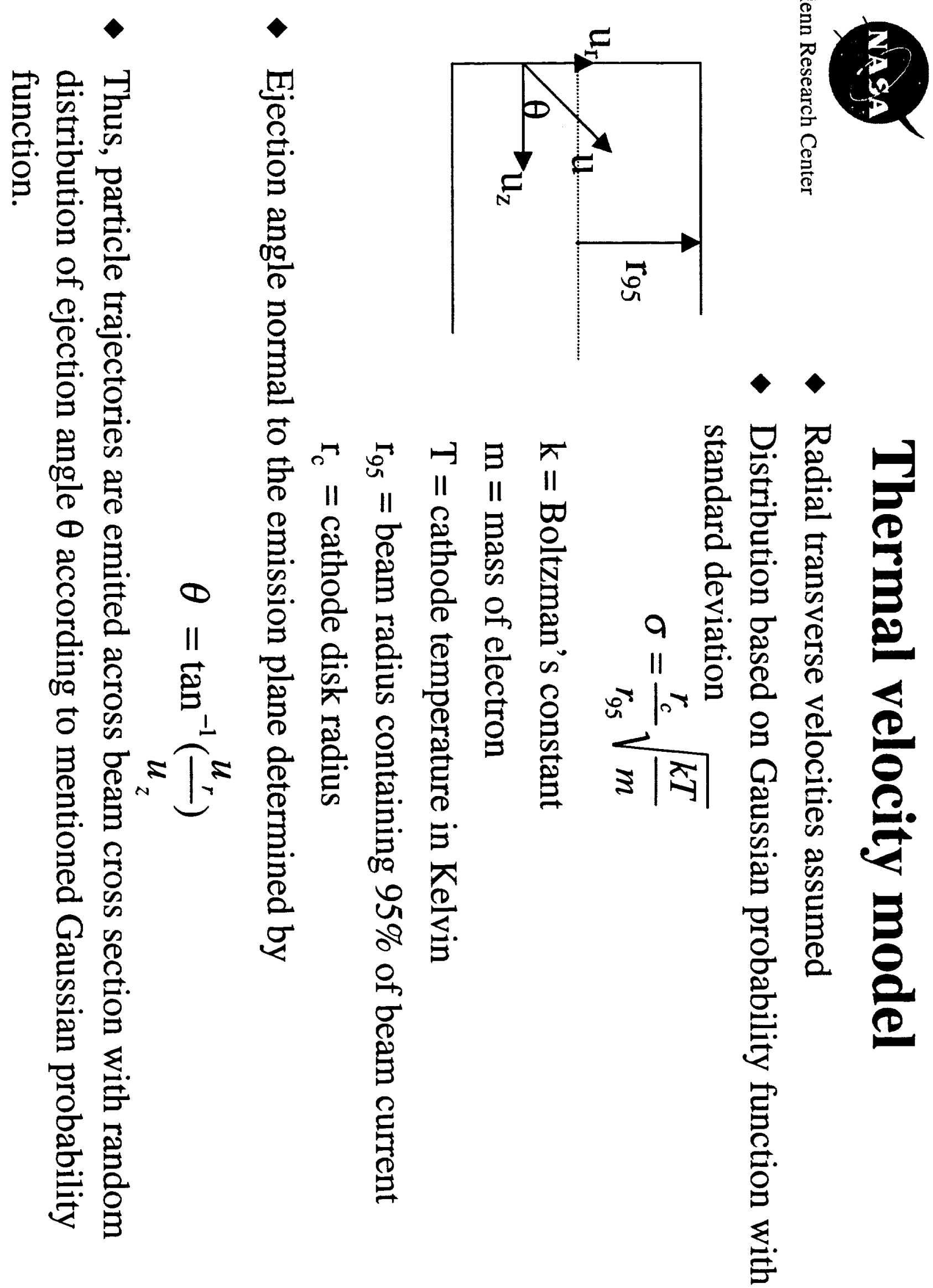



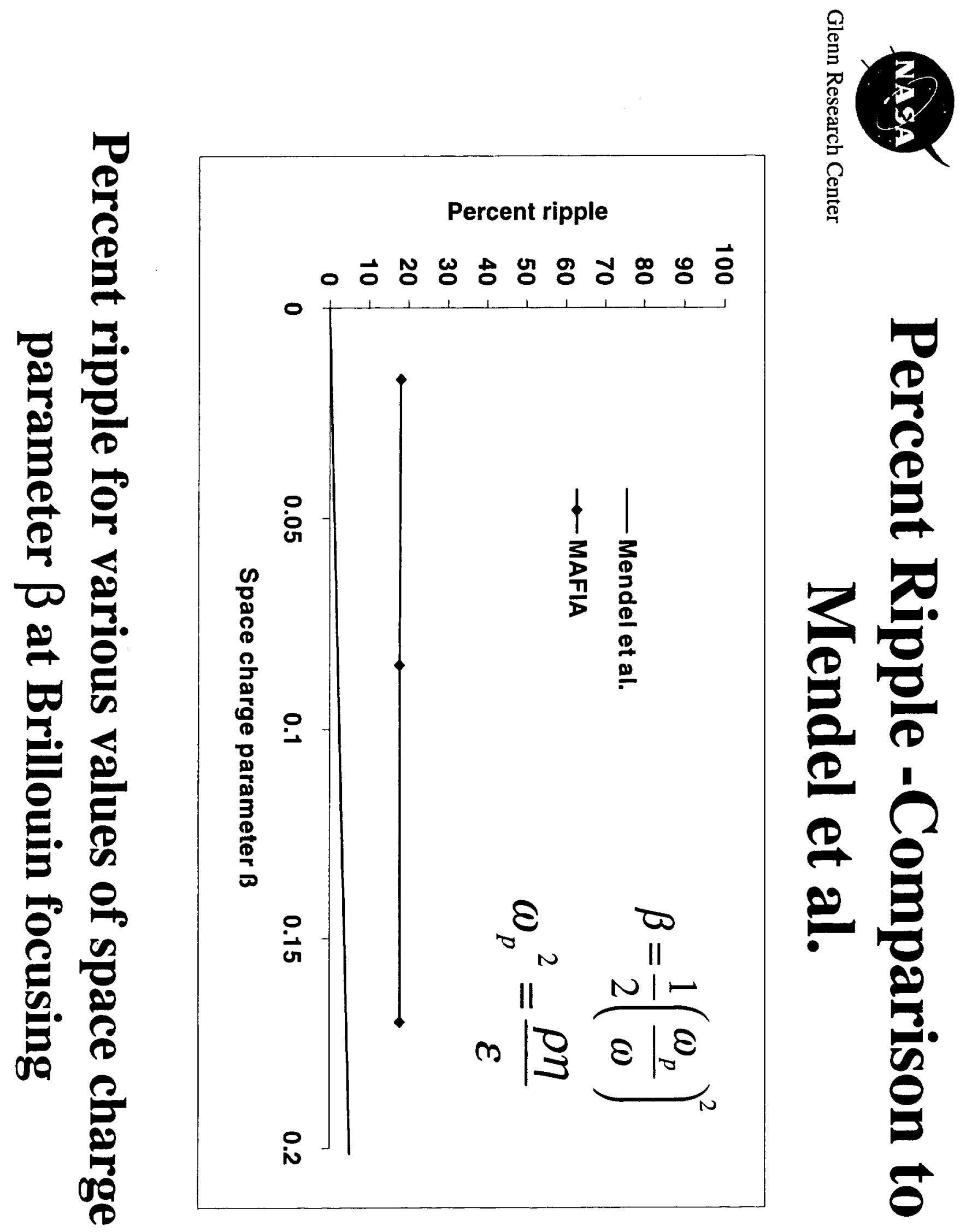


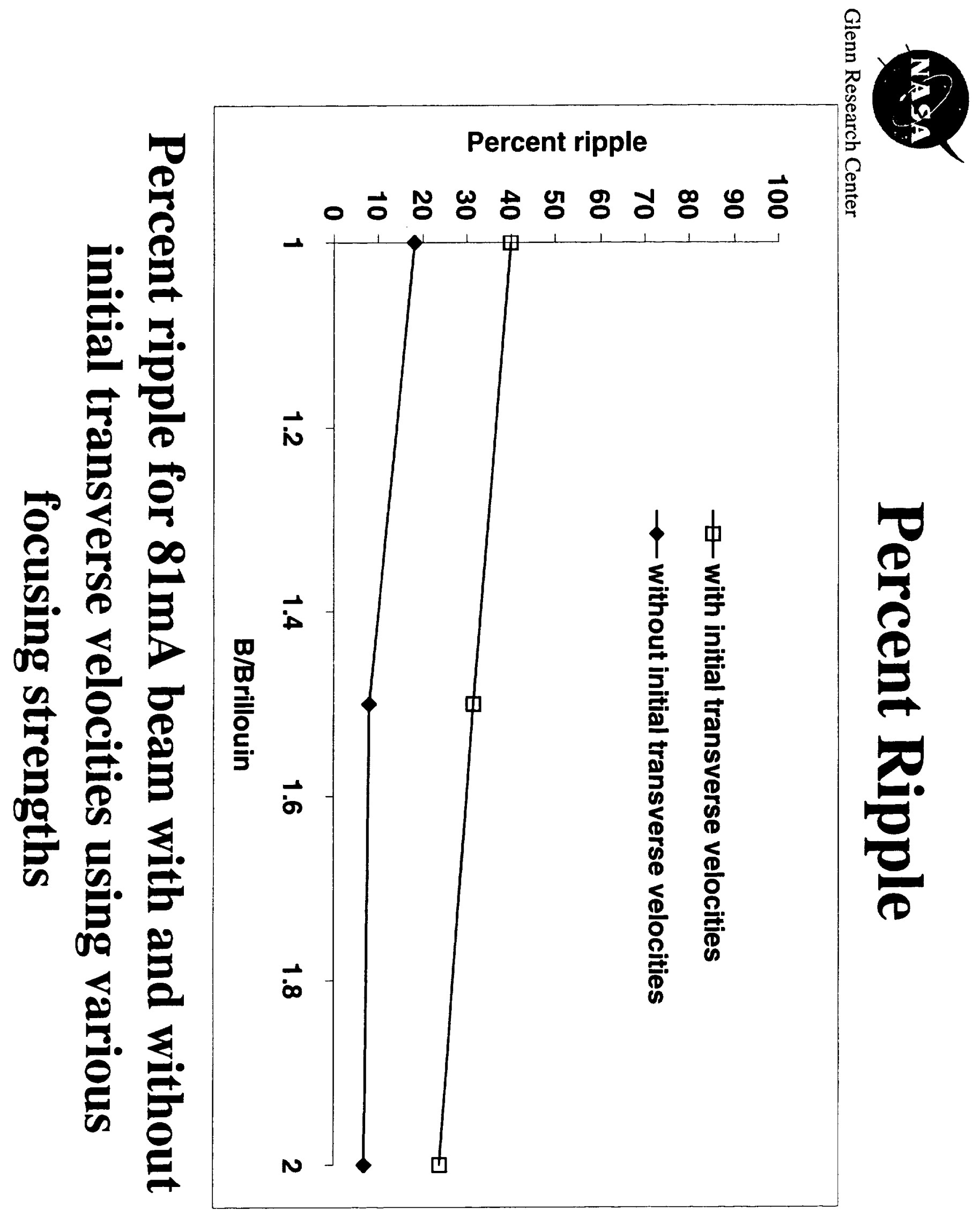




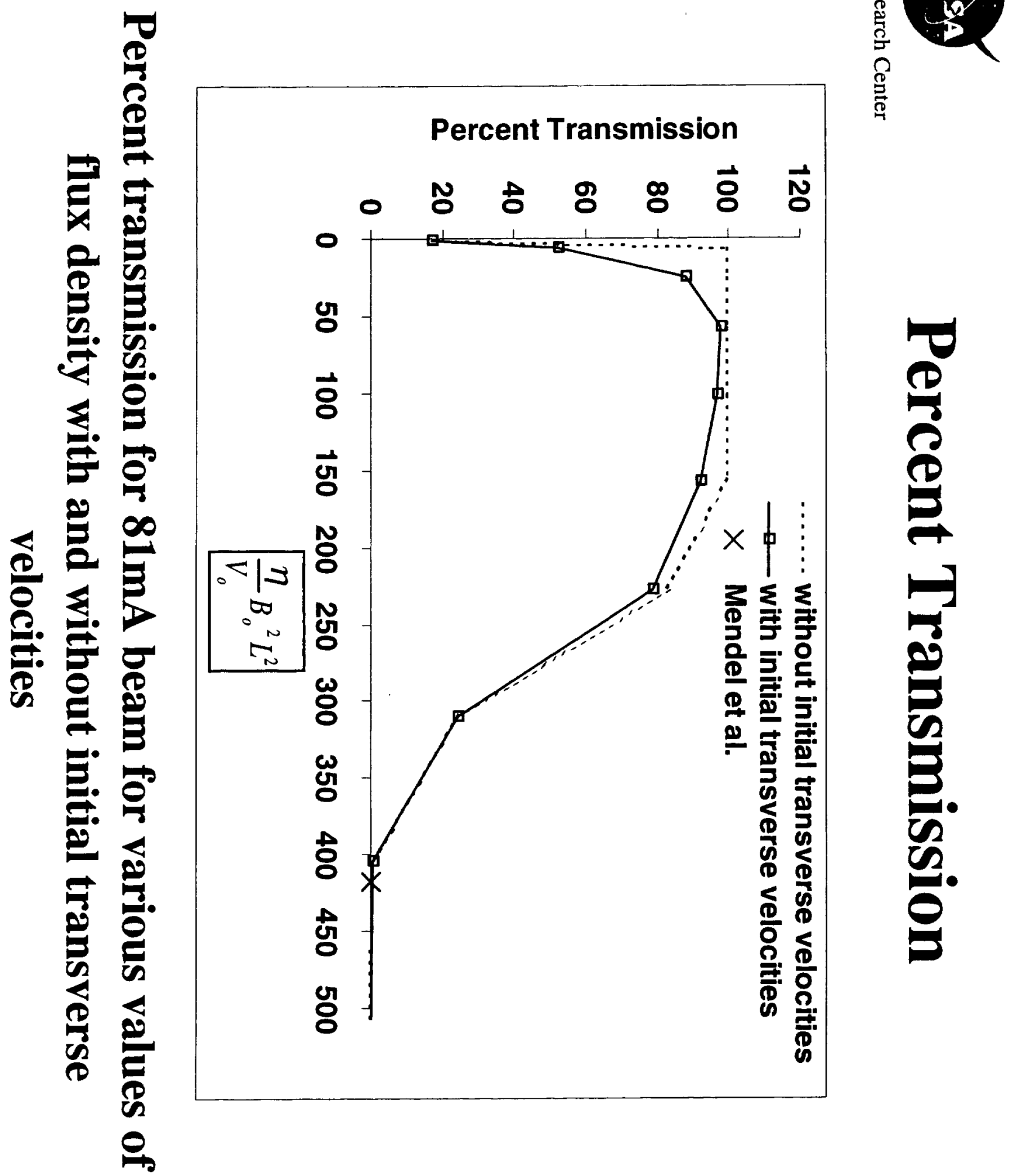




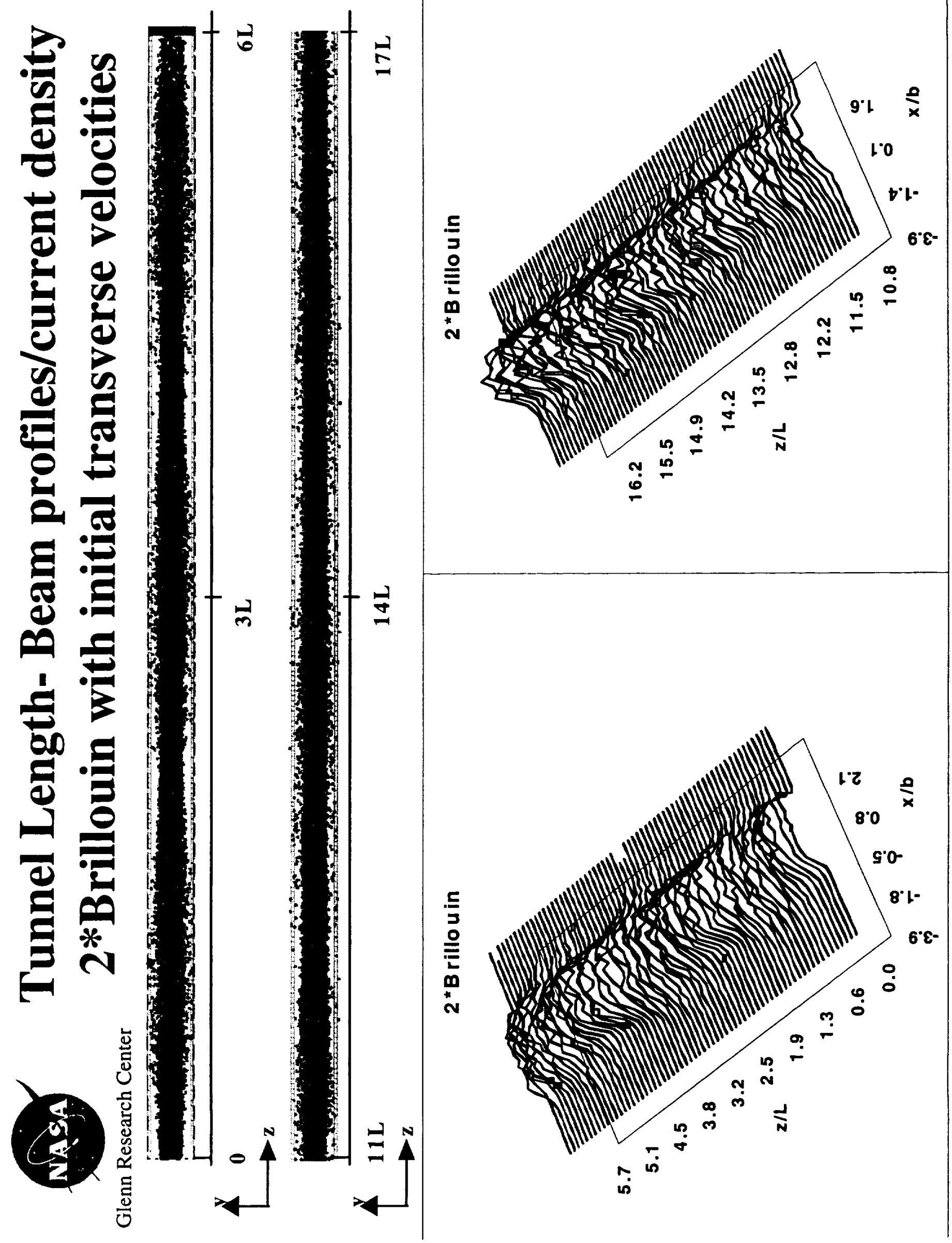




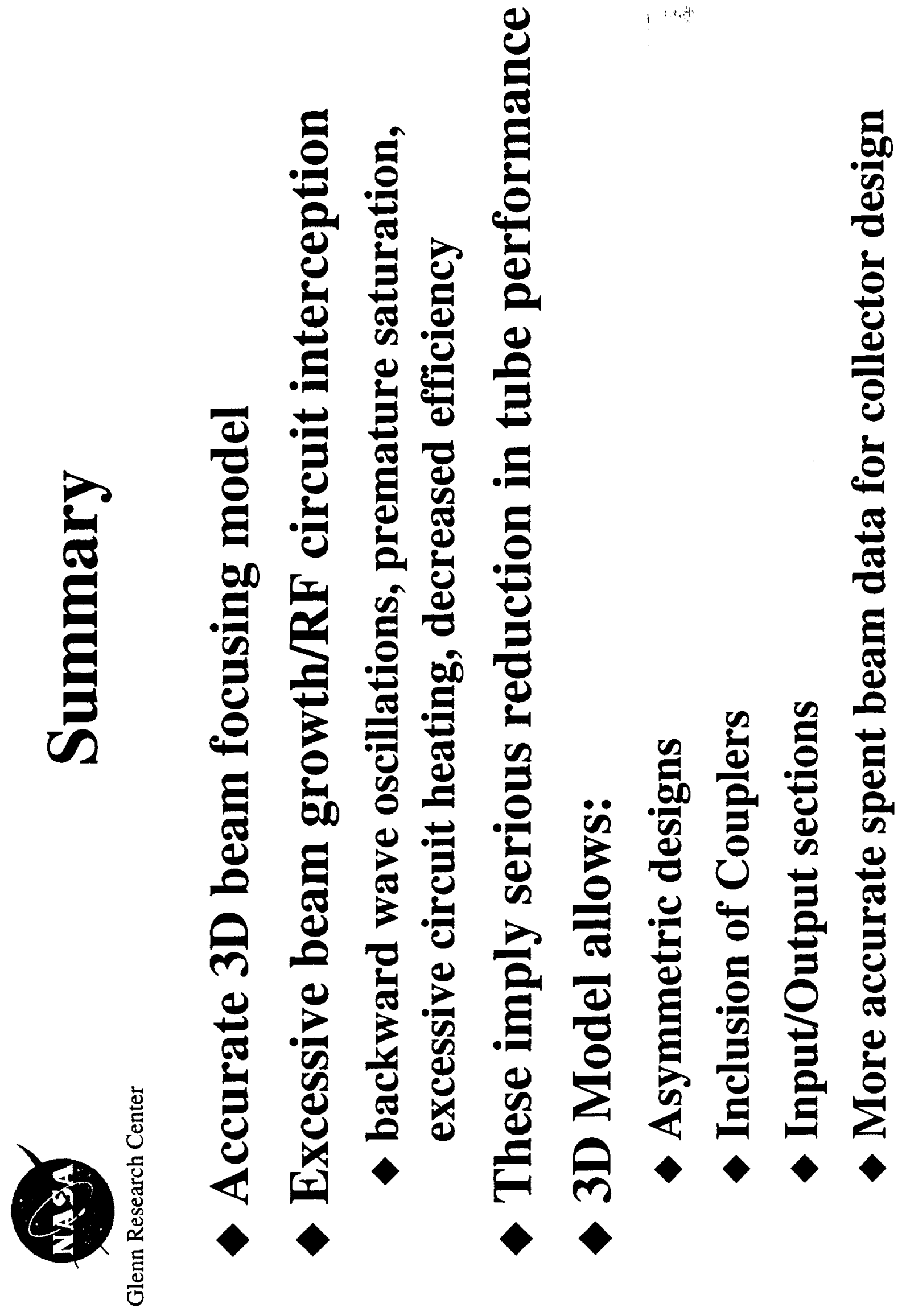


